\title{
AZUMAYA OBJECTS IN TRIANGULATED BICATEGORIES
}

\author{
NILES JOHNSON
}

\begin{abstract}
We introduce the notion of Azumaya object in general homotopy-theoretic settings. We give a self-contained account of Azumaya objects and Brauer groups in bicategorical contexts, generalizing the Brauer group of a commutative ring. We go on to describe triangulated bicategories and prove a characterization theorem for Azumaya objects therein. This theory applies to give a homotopical Brauer group for derived categories of rings and ring spectra. We show that the homotopical Brauer group of an Eilenberg-Mac Lane spectrum is isomorphic to the homotopical Brauer group of its underlying commutative ring. We also discuss tilting theory as an application of invertibility in triangulated bicategories.
\end{abstract}

\section{INTRODUCTION}

The notion of Azumaya algebra over a commutative ring $k$ generalizes the notion of a central simple algebra over a field. This was introduced by Azumaya Azu51] in the case that $k$ is local and by Auslander-Goldman AG60 for general $k$. The main classification theorem of Azumaya algebras says that the set of Morita-equivalence-classes of Azumaya $k$-algebras is the maximal subgroup of the monoid formed by Morita-equivalence-classes of $k$-algebras under $\otimes_{k}$. This is the Brauer group of $k$, an invariant which carries interesting algebraic and geometric information.

1.1. Foundations of homotopical Brauer theory. This work aims to develop foundations for Brauer theory in homotopical settings. We consider Azumaya objects in closed autonomous symmetric monoidal bicategories, and in particular focus on the triangulated bicategories arising as homotopy bicategories of rings and ring spectra. (The term "closed" refers to the existence of internal homs, and "autonomous" refers to opposites such as opposite algebras. See Sections 3.3 and 3.4.)

For the presentation here, we have three audiences in mind: the algebraic audience, for whom Azumaya algebras and Brauer groups of commutative rings are quite familiar; the categorical audience, for whom bicategories and invertibility therein are quite familiar; and the topological audience for whom structured ring spectra and the homotopical algebra thereof are quite familiar. We expect few readers to be in the intersection of these three audiences, and thus have attempted to write for the union of their complements.

In Sections 1 and 2 we introduce and motivate our results in the discrete and homotopical cases with a minimum of bicategorical language. This includes a review of classical Brauer groups for commutative rings as a special case of our general approach. A survey of the relevant bicategorical background is then presented in Section 3, with the general characterization of Azumaya objects in Section 3.6. We develop the notion of Azumaya object for triangulated bicategories in Section 4, and discuss homotopical (derived) applications in Section 5 .

Date: March 19, 2013.

2000 Mathematics Subject Classification. 55U99,18D35,16K50,14F22. 
Now we state the main definitions and results.

Definition 1.1 (Eilenberg-Watts Equivalence). Let $A$ and $B$ be a 0-cells of a bicategory $\mathscr{B}$. We say $A$ is Eilenberg-Watts equivalent to $B$ if there exists an invertible 1-cell $T: A \rightarrow B$. In bicategorical literature, this is known simply as equivalence of 0 -cells, but we prefer the more expressive term for this work.

Definition 1.2 (Eilenberg-Watts Bicategory). We say that a bicategory is Eilenberg-Watts if it is closed (i.e., has internal homs); has a symmetric monoidal product; and has an autonomous structure (i.e., a notion of "opposite" objects). Each of these bicategorical structures is described in Section 3 .

Definition 1.3 (Brauer Group, Azumaya Objects). Let $\mathscr{B}$ be a monoidal bicategory with unit 0-cell $k$. The Brauer group of $\mathscr{B}$, denoted $\operatorname{Br}(\mathscr{B})$, is the group of 1-cell equivalence classes (EilenbergWatts equivalence classes) of 0 -cells $A$ for which there exists a 0 -cell $B$ such that $A \otimes_{k} B$ is EilenbergWatts equivalent to $k$. Such 0 -cells are called Azumaya objects.

Remark 1.4. What we have termed "Eilenberg-Watts equivalence" is sometimes called "Morita equivalence", or sometimes "standard Morita equivalence" [KZ98]. The term "Morita equivalence" is also used frequently for monoid objects to mean that their categories of modules are equivalent. The fundamental theorem of Morita theory states that these notions coincide for algebras over a commutative ring and their module categories. Moreover, Rickard [Ric89] has shown that these notions coincide for algebras over a commutative ring and their derived categories of modules. However it is known that this coincidence does not generalize to derived categories of differential graded algebras or ring spectra [Shi06]. Work of Dugger and Dugger-Shipley analyzes the situation for differential graded algebras and ring spectra; see DS07b, DS07a and their related articles. The survey Joh08 compares the two notions abstractly for closed bicategories.

We have elected to introduce new terminology in hopes of clarifying the distinction between the two notions. See [Hov09] for a discussion of Eilenberg-Watts theorems in various settings.

The classical treatment of Azumaya algebras depends heavily on localization arguments and reducing to the case of algebras over fields; one feature of recent work in this area is a treatment that is independent of ideal theory: by introducing a bicategorical context we are able to generalize and unify the basic theory of Azumaya algebras and Brauer groups, giving elementary formal proofs of the main classification theorems. Similar ideas have appeared in algebraic and categorical literature - notably [Par75, Vit96, VOZ98, BV02]. We extend this approach to homotopical settings, giving a classification of Azumaya objects via invertibility in triangulated bicategories in Section 4.2. During preparation of this article, related work has appeared in [BRS12, Toë12, AG12, DT12].

Notation 1.5. Throughout, $k$ will denote the unit of a symmetric monoidal bicategory. In our applications, $k$ will often be a commutative $\left(E_{\infty}\right)$ d.g. algebra or ring spectrum. We let $\mathscr{C}_{k}$ denote the category of $k$-modules, noting that this is a symmetric monoidal category. We let $\mathscr{M}_{k}$ denote the bicategory of $k$-algebras and bimodules.

When $\mathscr{C}_{k}$ has a suitable model structure (described in Section 4.1, following [SS00]), then there is an induced model structure on $\mathscr{M}_{k}(A, B)$ for $k$-algebras $A$ and $B$. We let $\mathscr{D}_{k}(A, B)$ denote the corresponding homotopy category.

Taking as 0-cells the collection of $k$-algebras which are cofibrant as $k$-modules, we give one set of conditions in Proposition 4.14 which guarantee that $\mathscr{D}_{k}$ forms a triangulated bicategory with the additional Eilenberg-Watts structure necessary for the homotopical Brauer theory of Sections 3.6 and 4.2 . 
Although the bicategorical definitions of Azumaya object and Brauer group require only a monoidal structure, these are somewhat too general to be of practical interest. In an Eilenberg-Watts bicategory, we have characterization theorems for Azumaya objects in more concrete terms. In Definitions 3.10 and 3.11 we generalize the classical notions of central, (faithfully) separable, and faithfully projective $k$-algebras to Eilenberg-Watts bicategories. We also introduce the term "endomorphic", formally dual to the notion of centrality. We then show that these are the correct generalizations by proving the following theorem in Section 3.6:

Theorem 1.6. Let $A$ be a 0-cell of an Eilenberg-Watts bicategory, and let

$$
\lfloor A\rfloor: k \rightarrow A^{e}=A \otimes A^{\text {op }}
$$

denote the 1-cell induced by $A$ and the autonomous structure. The following are equivalent:

i. $\lfloor A\rfloor$ is an invertible 1 -cell.

ii. A is central and faithfully separable over $k$.

iii. A is endomorphic and faithfully projective over $k$.

iv. $A^{e}$ is Eilenberg-Watts equivalent to $k$.

$v$. There is a 0-cell $B$ such that $A \otimes B$ is Eilenberg-Watts equivalent to $k$.

Remark 1.7. The equivalence of the first three conditions is formal and straightforward. The argument proceeds by explaining that each of the second two conditions is an alternate description of invertibility. It is clear that the first condition implies the last two; one goal of this work is to develop a setting in which the reverse implications are also straightforward.

Another goal of this work is to prove a further characterization of Azumaya objects when (as in the derived and homotopical cases) the ambient bicategory carries a triangulated structure. In this case, the notion of invertibility admits alternate descriptions in terms of localization. This has been used in the topological case by Baker-Lazarev [BL04] for THH calculations. In Section 4 we introduce triangulated bicategories and prove a further characterization of Azumaya objects in that context. As with the rest of this theory, the notion of localization has both left (source) and right (target) versions, but we leave these definitions until Section 4 where we will develop more of the underlying bicategorical structure. The following is immediate from Proposition 4.21:

Theorem 1.8. Let A be a 0-cell in a triangulated Eilenberg-Watts bicategory. The following statements are equivalent:

i. A is an Azumaya object.

ii. $A$ is dualizable over $A^{e}$ and central over $k$, and $A^{e}$ is strongly (target-)A-local.

iii. $A$ is dualizable over $k$ and endomorphic over $k$, and $k$ is strongly (source-)A-local.

Using these general characterizations of Azumaya objects, we show in Section 5 that the homotopical Brauer groups defined here agree with those of [Toë12] for derived categories of rings and of [BRS12] for commutative $S$-algebras.

1.2. Brauer groups of Eilenberg-Mac Lane spectra. One application of the foundations developed here is an immediate computation of the Brauer group of an Eilenberg-Mac Lane spectrum. We include the proof because it is short and because it illustrates the utility of the setting we develop: that which should be formal (because the homotopical algebra of Eilenberg-Mac Lane spectra is equivalent to the ordinary algebra of their underlying rings) has become straightforward (because the Eilenberg-Mac Lane functor induces a local equivalence of triangulated bicategories). 
Theorem 1.9. Let $k$ be a commutative ring. The Eilenberg-Mac Lane functor to symmetric spectra

$$
H: \mathscr{C}_{k} \rightarrow \mathscr{C}_{H k}
$$

induces an isomorphism of Brauer groups

$$
H: \operatorname{Br}\left(\mathscr{D}_{k}\right) \rightarrow \operatorname{Br}\left(\mathscr{D}_{H k}\right) .
$$

Proof. By Remark 4.5 and Proposition 4.15 we have triangulated Eilenberg-Watts bicategories $\mathscr{D}_{k}$ and $\mathscr{D}_{H k}$. The Eilenberg-Mac Lane functor $H$ is a weak monoidal Quillen equivalence, which means that $H$ is a lax monoidal functor (but its adjoint is not necessarily monoidal). Thus $H$ sends differential graded $k$-algebras to $H k$-algebras, and sends $(A, B)$-bimodules to $(H A, H B)$-bimodules [Shi07. We have natural equivalences in $\mathscr{D}_{H k}(H C, H B)$

$$
H\left(X \wedge_{A} W\right) \simeq H X \wedge_{H A} H W
$$

for $X \in \mathscr{D}_{k}(C, A)$ and $Y \in \mathscr{D}_{k}(A, B)$. Therefore $H$ induces a pseudofunctor

$$
\mathscr{D}_{k} \rightarrow \mathscr{D}_{H k} .
$$

Moreover, it gives an equivalence

$$
\mathscr{D}_{k}(A, B) \stackrel{\simeq}{\rightarrow} \mathscr{D}_{H k}(H A, H B)
$$

for all differential graded $k$-algebras $A$ and $B$ and hence a bijection on isomorphism classes of invertible bimodules and on Eilenberg-Watts equivalence classes of Azumaya objects. Thus we have an isomorphism of Brauer groups.

Remark 1.10. Note that this result depends on the base commutative ring spectrum $H k$ being Eilenberg-Mac Lane. If $H k$ is an algebra over another commutative ring spectrum $R$, then restriction along the unit map $R \rightarrow H k$ yields a pseudofunctor of bicategories and hence a map of Brauer groups

$$
\operatorname{Br}\left(\mathscr{D}_{k}\right) \stackrel{\cong}{\rightrightarrows} \operatorname{Br}\left(\mathscr{D}_{H k}\right) \rightarrow \operatorname{Br}\left(\mathscr{D}_{R}\right)
$$

which may be neither injective nor surjective. The interesting example of [Shi06, §5] shows that restriction from $H \mathbb{Z}$ to the sphere spectrum is non-injective on Eilenberg-Watts equivalence classes of $H \mathbb{Z}$-algebras.

As an immediate corollary, we have a computation of the Brauer group for an Eilenberg-Mac Lane spectrum of a field in terms of the classical Brauer group:

Corollary 1.11. If $k$ is a field, then $\operatorname{Br}\left(\mathscr{D}_{H k}\right) \cong \operatorname{Br}(k)$. In particular, $\operatorname{Br}\left(\mathscr{D}_{H k}\right)=0$ if $k$ is finite or algebraically closed.

Proof. Proposition 5.3 says that $\operatorname{Br}\left(\mathscr{D}_{k}\right) \cong B r(k)$. The result then follows from Theorem 1.9.

In the case that $k$ is an algebraically closed field, this was established by BRS12] using different methods, but also relying on [Toë12] for the comparison with the derived Brauer group of a field.

1.3. Invertibility. Sections 3.5 and 4.2 give a general study of invertibility in triangulated bicategories, and this forms the foundation of the characterization theorems. Proposition 4.21 gives a general characterization of invertible objects in triangulated bicategories. As further applications, we recover results from the derived Morita (Eilenberg-Watts) theory of Rickard [Ric89] and Schwede-Shipley [SS03b]. The object $T$ here is called a tilting object. 
Proposition 1.12 ([Ric89]). Let $k$ be a commutative ring, and let $\mathscr{D}_{k}$ denote the bicategory of $k$-algebras and derived categories of bimodules. Suppose $B$ is a differential graded $k$-algebra, and let $T$ be a differential graded right $B$-module. Let $A=\operatorname{End}_{B}(T)$ be the endomorphism algebra of $T$. If $T$ has the following two properties, then $\mathscr{D}_{k}(B)$ and $\mathscr{D}_{k}(A)$ are equivalent as triangulated categories.

i. $T$ is a right-dualizable $B$-module.

ii. $T$ generates the triangulated category $\mathscr{D}_{k}(B)$.

Note. Since $T$ is right-dualizable, $T$ is a bounded complex of finitely-generated and projective $B$-modules. Therefore the derived and underived endomorphism algebras are equal.

Proposition 1.13 ( $[\mathrm{SS03b})$. Let $k$ be a commutative ring spectrum, and let $\mathscr{D}_{k}$ denote the bicategory of $k$-algebras and homotopy categories of bimodules. Suppose $B$ is a $k$-algebra, and let $T$ be a right $B$-module. Let $A=F_{B}(T, T)$ be the endomorphism ring spectrum of $T$. If $T$ has the following two properties, then $\mathscr{D}_{k}(B)$ and $\mathscr{D}_{k}(A)$ are equivalent categories.

i. $T$ is right-dualizable as a B-module.

ii. $T$ generates the triangulated category $\mathscr{D}_{k}(B)$.

1.4. Acknowledgements. This work developed from a portion of the author's Ph.D. thesis. The author thanks his advisor, Peter May, for reading it many times in draft form and offering a number of suggestions both mathematical and stylistic. The author also thanks Michael Ching for his significant encouragement.

The exposition has benefited from the helpful suggestions of anonymous referees, and from the audiences who have listened to talks and read drafts of this article. The terminology "EilenbergWatts equivalence" was suggested by Justin Noel during one of many helpful conversations.

\section{Motivation}

To help motivate the generality of Sections 3 and 4 needed for Theorems 1.6 and 1.8 , we give parallel introductions of the classification theorems for classical algebra and for homotopical settings (Sections 2.1 and 2.2, respectively). Both are special cases of the general theory. In Example 2.7 we sketch a number of additional examples to which the theory applies.

2.1. Brauer groups of discrete rings. In this section we review the classical theory of Brauer groups for commutative (discrete) rings. Let $k$ be a commutative ring and let $\mathscr{B}=\mathscr{M}_{k}$ be the bicategory of $k$-algebras and their bimodules. A 0 -cell $A$ of this bicategory is a $k$-algebra, and $A^{e}$ is the enveloping $k$-algebra $A \otimes_{k} A^{\text {op }}$. Note that throughout this section we regard $A$ as a right module over $A^{e}$ and a left module over $k$. We recall the classical characterization/definition of Azumaya 
algebras after recalling basic terminology and a well-known lemma. These are special cases of the more general terms introduced in Definitions 3.10 and 3.11 .

\section{Definitions 2.1.}

- $A$ is called separable over $k$ if $A$ is projective as a module over $A^{e}$. Since $A$ is always finitely generated over $A^{e}$, this is equivalent to the condition that $A$ be a dualizable module over $A^{e}$. By the dual basis lemma, this is equivalent to the condition that the coevaluation

$$
A \otimes_{A^{e}} \operatorname{Hom}_{A^{e}}\left(A, A^{e}\right) \rightarrow \operatorname{Hom}_{A^{e}}(A, A)
$$

be an isomorphism. To motivate this term, it should be noted that when $k$ is a field, $A$ being separable over $k$ implies that $A$ is semi-simple over $k$ and remains semi-simple upon extension of scalars over any field extension of $k$. When $A$ is also a field, this implies that $A$ is a separable extension of $k$ in the usual sense for fields [Coh03.

- $A$ is called central over $k$ if the center of $A$ is precisely $k$; this occurs if and only if the unit

$$
k \rightarrow \operatorname{Hom}_{A^{e}}(A, A)
$$

is an isomorphism.

- $A$ is called faithfully projective over $k$ if both the coevaluation

$$
\operatorname{Hom}_{k}(A, k) \otimes A \rightarrow \operatorname{Hom}_{k}(A, A)
$$

and the evaluation

$$
A \otimes_{A^{e}} \operatorname{Hom}_{k}(A, k) \rightarrow k
$$

are isomorphisms. Note, again by the dual basis lemma, that the coevaluation being an isomorphism is equivalent to $A$ being finitely-generated and projective as a $k$-module. The evaluation map being an isomorphism implies that $-\otimes_{k} A$ is object faithful, meaning that $M \otimes_{k} A=0$ implies $M=0$.

Lemma 2.2 (See e.g. [KO74] or [DI71]). If $A$ is central and separable over $k$, then the evaluation

$$
\operatorname{Hom}_{A^{e}}\left(A, A^{e}\right) \otimes_{k} A \rightarrow A^{e}
$$

is an isomorphism.

Here is the classical theorem, which follows directly from Theorem 1.6. It provides a link between Eilenberg-Watts equivalences and the more calculable conditions of central, separable and faithfully projective.

Theorem (See 1.6). Let $k$ be a commutative ring, and $A$ a k-algebra. The following are equivalent:

i. A is invertible as a $\left(k, A^{e}\right)$-bimodule, thus providing an Eilenberg-Watts equivalence between $A^{e}$ and $k$.

ii. A is central and separable over $k$.

iii. A is faithfully projective over $k$ and the unit map

$$
A^{e} \rightarrow \operatorname{Hom}_{k}(A, A)
$$

is an isomorphism.

$i v$. There is a $k$-algebra $B$ such that $A \otimes_{k} B$ is Eilenberg-Watts equivalent to $k$.

Remark 2.3. Note that the last condition of Item iii means that every $k$-linear endomorphism of $A$ is given by left and right multiplication in $A$. We call such an algebra "endomorphic" in Definition 2.4. 
2.2. Homotopical Brauer groups. In Section 4.1 we describe a bicategorical structure on the collection of categories $\mathscr{D}_{k}(A, B)$, where $k$ is a commutative $\left(E_{\infty}\right)$ d.g. algebra or ring spectrum, cofibrant as a module over itself, and $A$ and $B$ are cofibrant as $k$-modules (see Proposition 4.15). This consists mainly of assembling the necessary results from the literature on monoidal model categories, but we have presented a careful treatment of the Eilenberg-Watts structure.

In this section we continue to motivate the general theory by describing the definitions and Azumaya characterization theorem in this special case. We use $\wedge$ to denote the derived tensor product, and $F$ to denote the derived hom. The isomorphisms in the homotopy category are the weak equivalences, and so we switch to this terminology. Let $k$ be a commutative ring, and let $A$ be a differential-graded algebra over $k$. For the topologically inclined reader, $k$ and $A$ can be taken to be structured ring spectra - the modern foundations of spectra such as [EKMM97, MMSS01, allow us to treat the algebraic and topological cases with the same categorical arguments. For either the algebraic or topological cases, we assume throughout that $k$ and $A$ are cofibrant with respect to a given model structure on $\mathscr{C}_{k}$.

To begin, we make the following definitions inspired by the classical case. They are again special cases of the general bicategorical definitions in 3.10 and 3.11 .

Definition 2.4 (Central/Endomorphic). We say that $A$ is central if the natural unit map

$$
k \rightarrow F_{A^{e}}(A, A)
$$

is a weak equivalence. Note that $F_{A^{e}}(A, A)$ is the (topological) Hochschild cohomology of $A$ over $k$.

We say that $A$ is endomorphic if the natural unit map from $A^{e}$ to the endomorphism algebra

$$
A^{e} \rightarrow F_{k}(A, A)
$$

is a weak equivalence.

Definition 2.5 (Faithfully Projective/Faithfully Separable). We say that $A$ is faithfully projective over $k$ if both the coevaluation

$$
F_{k}(A, k) \wedge_{k} A \rightarrow F_{k}(A, A)
$$

and the evaluation

$$
A \wedge_{A^{e}} F_{k}(A, k) \rightarrow k
$$

are weak equivalences. Note that the first of these two conditions is equivalent to requiring that $A$ be dualizable (in the derived category) over $k$. (Duality is discussed in Section 3.5.) The second of these two conditions implies a kind of faithfulness over $k$, as in the classical case.

We say that $A$ is faithfully separable over $k$ if both the coevaluation

$$
A \wedge_{A^{e}} F_{A^{e}}\left(A, A^{e}\right) \rightarrow F_{A^{e}}(A, A)
$$

and the evaluation

$$
F_{A^{e}}\left(A, A^{e}\right) \wedge_{k} A \rightarrow A^{e}
$$

are weak equivalences. The first of these two conditions is equivalent to requiring that $A$ be dualizable over $A^{e}$, and the second is a kind of faithfulness over $A^{e}$.

Definition 2.6. We call $A$ an Azumaya object if any of the equivalent conditions below hold.

Theorem (See 1.6). The following statements are equivalent:

i. $A$ is an invertible bimodule.

ii. A is central and faithfully separable over $k$. 
iii. A is endomorphic and faithfully projective over $k$.

iv. $A^{e}$ is Eilenberg-Watts equivalent to $k$.

$v$. There exists a $k$-algebra $B$ such that $A \wedge_{k} B$ is Eilenberg-Watts equivalent to $k$.

2.3. Additional Examples. For concreteness, we focus our main applications on the EilenbergWatts bicategories coming from differential graded algebras or from ring spectra. But the bicategorical setting is quite general and here we sketch several additional examples to which the theory applies.

Example 2.7 (Symmetric monoidal categories). The example of rings and modules motivates a standard construction for a bicategory $\mathscr{M}_{\mathscr{C}}$ from any complete and cocomplete closed symmetric monoidal category $\mathscr{C}$. The 0 -cells are taken to be the monoids of $\mathscr{C}$, and 1-cells are taken to be bimodules. Bimodule maps are defined just as in the case of bimodules over rings, and these constitute the 2-cells of $\mathscr{M}_{\mathscr{C}}$. Colimits from $\mathscr{C}$ are used to construct the horizontal composition in $\mathscr{M}_{\mathscr{C}}$, in the same way that coequalizers give the tensor product of two modules over a ring. Limits give $\mathscr{M}_{\mathscr{C}}$ a closed structure in the same way that equalizers give the homomorphisms of two modules over a ring.

Remembering the underlying symmetric monoidal product of $\mathscr{C}$ gives $\mathscr{M}_{\mathscr{C}}$ an autonomous symmetric monoidal structure, and $\mathscr{M}_{\mathscr{C}}$ is therefore Eilenberg-Watts. Theorem 1.6 thus characterizes the Azumaya monoids of $\mathscr{C}$, and defines a Brauer group of $\mathscr{C}$ which reduces to the Brauer group of $k$ when $\mathscr{C}$ is the symmetric monoidal category of modules over a commutative ring $k$.

The Brauer group of a symmetric monoidal category has been defined directly by Pareigis [Par75] and, for a braided monoidal category, by Van Oystaeyen-Zhang [VOZ98]. These definitions (in the symmetric monoidal case) are equivalent to ours, but there the emphasis is on other aspects of the classical theory (e.g. separability and Casimir elements); their work does not give the full characterization of Azumaya algebras presented in Theorem 1.6. Likewise, we do not discuss the variants on Brauer theory described by Pareigis or Van Oystaeyen-Zhang. Vitale [Vit96] gives a thorough treatment of Brauer groups for symmetric monoidal categories, and a pleasant summary of categorical approaches to this case.

Example 2.8 (Ex-spaces or parametrized spectra). May-Sigurdsson introduce $\mathscr{E} x$, the bicategory of parametrized spectra in [MS06, 17.1.3]. The 0-cells of $\mathscr{E} x$ are topological spaces, and the category of 1-cells $\mathscr{E} x(A, B)=H o \mathscr{S}_{A \times B}$ is the homotopy category of parametrized spectra over $A \times B$. They describe a natural horizontal composition

$$
\odot: \mathscr{E} x(A, B) \times \mathscr{E} x(B, C) \rightarrow \mathscr{E} x(A, C)
$$

by pullback along the diagonal $A \times B \times C \rightarrow A \times B \times B \times C$ and pushforward along the projection $A \times B \times C \rightarrow A \times C$. These have adjoints given by pushforward and pullback along the same maps, and this defines a closed structure adjoint to the horizontal composition.

The external smash product

$$
\bar{\wedge}: \operatorname{Ho} \mathscr{S}_{A} \times \operatorname{Ho} \mathscr{S}_{A^{\prime}} \rightarrow \operatorname{Ho} \mathscr{S}_{A \times A^{\prime}}
$$

gives $\mathscr{E} x$ a symmetric monoidal structure. The "opposite" of a space $A$ is again $A$, and the equivalence $\mathscr{E} x(A, B) \simeq \mathscr{E} x\left(B^{\text {op }}, A^{\text {op }}\right)=\mathscr{E} x(B, A)$ is induced by the switch map

$$
t: A \times B \rightarrow B \times A .
$$

Thus $\mathscr{E} x$ is an Eilenberg-Watts bicategory and the characterization of Azumaya objects in $\mathscr{E} x$ follows as in the case of spectra. 
Example 2.9 (Rings with many objects). Let $\mathcal{A}$ be a small category enriched in $\mathcal{A} 6$, the category of abelian groups. A left $\mathcal{A}$-module is a functor $\mathcal{A} \rightarrow \mathscr{A} b$, and a right module is a functor $\mathcal{A}^{\text {op }} \rightarrow \mathcal{A} \mathfrak{b}$; morphisms of $\mathcal{A}$-modules are enriched natural transformations. Now let $\mathscr{R}$ ingoids be the bicategory whose 0 -cells are small $\mathscr{A} 6$-categories, 1-cells are bimodules, and 2-cells are natural transformations. The tensor product and hom for bimodules induce horizontal composition with a closed structure, and the tensor product in $\mathscr{A} 6$ induces a symmetric monoidal product on $\mathscr{R}$ ingoids making it an Eilenberg-Watts bicategory. The unit object is the one-object category whose endomorphism ring is the ring of integers.

Clearly this construction may be generalized by replacing $\mathcal{A} 6$ with a bicomplete closed symmetric monoidal category $\mathbf{V}$. We denote the bicategory so constructed by $\mathbf{V}-\mathscr{R}$ ingoids. This too is an Eilenberg-Watts bicategory, and thus Theorem 1.6 gives a characterization of Azumaya objects.

In the case that $\mathbf{V}=\operatorname{Mod}_{k}$ is the category of modules over a commutative ring $k$, there is a natural morphism of bicategories

$$
\iota: \mathscr{M}_{k} \rightarrow \operatorname{Mod}_{k}-\mathscr{R} \text { ingoids }
$$

given by sending a $k$-algebra $A$ to the corresponding 1-object $\operatorname{Mod}_{k}$-category, sending an $(A, B)$ bimodule to the functor whose value on the single object of $\iota(A) \times \iota(B)^{\mathrm{op}}$ is the given bimodule, and sending a morphism of bimodules to the induced natural transformation. The unit object is $\iota(k)$, the one-object category with endomorphism ring $k$. The morphism $\iota$ preserves the Azumaya property, and induces a homomorphism of Brauer groups

$$
\operatorname{Br}\left(\mathscr{M}_{k}\right) \rightarrow \operatorname{Br}\left(\operatorname{Mod}_{k}-\mathscr{R} \text { ingoids }\right) \text {. }
$$

For each pair of $k$-algebras, $A$ and $B$, the functor $\iota: \mathscr{M}_{k}(A, B) \rightarrow \operatorname{Mod}_{k}-\mathscr{R}$ ingoids $(\iota A, \iota B)$ is an equivalence of categories and hence the induced map on Brauer groups is an injection. This map is in fact an isomorphism, as shown by [BV02, 5.7].

Example 2.10 (Sheaves). The Brauer group of a sheaf $\mathscr{O}$ of commutative rings was introduced by Auslander in Aus66 and by Grothendieck in Gro68. By Theorem 1.6 this is the same as the Brauer group of the symmetric monoidal category of $\mathscr{O}$-modules: a map of sheaves is an isomorphism if and only if it is so locally, and thus an Azumaya object in the bicategory of $\mathscr{O}$-algebras and their bimodules is precisely the same as a sheaf of Azumaya algebras. This perspective was initiated by Auslander's work, and has been discussed by Van Oystaeyen-Zhang [VOZ98, 3.5(2); 3.7].

\section{Eilenberg-Watts Bicategories}

Recall that we use the term "Eilenberg-Watts bicategory" for a closed autonomous symmetric monoidal bicategory. We describe these terms in Sections 3.1 to 3.4. Section 3.5 reviews duality and invertibility in bicategories. In Section 3.6 we prove the characterization theorem for Azumaya objects in Eilenberg-Watts bicategories.

Notation 3.1. We use arrows such as $f: M \rightarrow M^{\prime}$ to denote that $f$ is a 2-cell with source $M$ and target $M^{\prime}$, and slashed arrows such as $M: A \rightarrow B$ to denote that $M$ is a 1-cell with source $A$ and target $B$. We use $\circ$ or juxtaposition to denote vertical composition of 2-cells, and $\odot$ to denote horizontal composition of 1 -cells and of 2 -cells. We write $\odot$-composition in "diagrammatic order", so that a composite of 1-cells

$$
A \stackrel{M}{\rightarrow} B \stackrel{N}{\rightarrow} C
$$

is denoted $(M \odot N): A \rightarrow C$. 
3.1. Monoidal bicategories. A monoidal bicategory can be defined as a tricategory with one object, in the sense of GPS95. In practical terms, this means that the bicategory is equipped with an additional monoidal product on 0 -, 1-, and 2-cells forming a pseudofunctor

$$
\otimes: \mathscr{B} \times \mathscr{B} \rightarrow \mathscr{B} \text {. }
$$

For categories of 1-cells $A \rightarrow B$ and $A^{\prime} \rightarrow B^{\prime}$, this gives functors

$$
\otimes: \mathscr{B}(A, B) \times \mathscr{B}\left(A^{\prime}, B^{\prime}\right) \rightarrow \mathscr{B}\left(A \otimes A^{\prime}, B \otimes B^{\prime}\right) .
$$

For associativity and unit constraints we have invertible 1-cells

$$
a:\left(A_{1} \otimes A_{2}\right) \otimes A_{3} \rightarrow A_{1} \otimes\left(A_{2} \otimes A_{3}\right)
$$

and

$$
\begin{gathered}
l: A \otimes k \rightarrow A \\
r: k \otimes A \rightarrow A .
\end{gathered}
$$

Invertible 2-cells fill the associativity pentagon and unit triangle, and there are two additional invertible 2-cells relating the left and right units with the associativity constraint. This data satisfies a number of reasonable axioms which we will not need to reference explicitly.

In lieu of further details, which can be found in GPS95, Str03, SP09, we encourage the reader unfamiliar with monoidal bicategories to concentrate on the representative example of algebras and bimodules. Here the 0-cells are algebras over a commutative ring $k$, 1 -cells $A \rightarrow B$ are bimodules, and 2-cells are bimodule homomorphisms. The horizontal composition

$$
A \rightarrow B \rightarrow C
$$

is given by the tensor product of bimodules $\otimes_{B}$. The tensor product of algebras $\otimes_{k}$ gives a monoidal structure on this bicategory. This example contains all of the essential structure present in the more sophisticated algebraic and topological examples, and also serves to illustrate the additional bicategorical structure described below.

A trivial but slightly technical lemma about monoidal structure will be useful later:

Lemma 3.2. Let $k$ be the unit of a monoidal bicategory, and suppose that $M: C \rightarrow k$ and $N: k \rightarrow D$ are 1-cells. Then there are natural isomorphisms

$$
\begin{aligned}
& N \otimes M \cong M \odot N \\
& N \otimes M \cong(N \otimes C) \odot(D \otimes M)
\end{aligned}
$$

and hence there is a 2-cell isomorphism filling the square below:

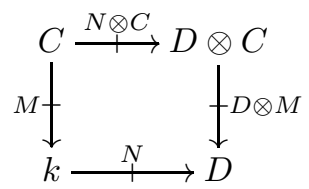

Proof. Pseudofunctorality of the monoidal product means we have isomorphisms

$$
(k \odot N) \otimes(M \odot k) \cong(k \otimes M) \odot(N \otimes k)
$$

and

$$
(N \odot D) \otimes(C \odot M) \cong(N \otimes C) \odot(D \otimes M) .
$$

Composing with unit isomorphisms then gives the result. 
3.2. Symmetric monoidal bicategories. Symmetry for the monoidal product in a bicategory entails invertible 1-cells

$$
R_{A B}: A \otimes B \rightarrow B \otimes A,
$$

and again the example of algebras and bimodules is illuminating. The structure 1-cells form a braiding and for each pair $(A, B)$ there is an invertible 2-cell (syllepsis) making the composite 1-cell

$$
R_{A B} \odot R_{B A}: A \otimes B \rightarrow B \otimes A \rightarrow A \otimes B
$$

isomorphic to the identity 1-cell. The symmetry for this syllepsis is an axiom equating the two isomorphisms $R_{A B} \odot R_{B A} \odot R_{A B} \cong R_{A B}$, one coming from the syllepsis for $(A, B)$ and the other coming from that of $(B, A)$. Descriptions of braided, sylleptic, and symmetric monoidal bicategories, together with coherence theorems in the braided and symmetric cases, are found in Gur11, GO12.

3.3. Autonomous structure. A symmetric monoidal bicategory $\mathscr{B}$ is autonomous [DS97] if each 0 -cell $A$ has an opposite, $A^{\text {op }}$, providing an involution on $\mathscr{B}$ and such that there are (suitably natural) equivalences of categories

$$
\mathscr{B}(A \otimes B, C) \simeq \mathscr{B}\left(B, A^{\text {op }} \otimes C\right) \quad \text { and } \quad \mathscr{B}\left(B \otimes A^{\text {op }}, C\right) \simeq \mathscr{B}(B, C \otimes A)
$$

for all $B$ and $C$. As in classical ring theory, where $A^{\text {op }}$ is the opposite $k$-algebra, we write $A^{e}$ for the enveloping object $A \otimes A^{\mathrm{op}}$, and given a 1-cell $M: A \rightarrow B$ we write $M^{\mathrm{op}}: B^{\mathrm{op}} \rightarrow A^{\mathrm{op}}$ for its image under the equivalence

$$
\mathscr{B}(A, B) \simeq \mathscr{B}\left(B^{\text {op }}, A^{\text {op }}\right)
$$

3.4. Closed structure. A closed structure for a bicategory, $\mathscr{B}$, defines right adjoints for $\odot$. For a 1-cell $M$, the right adjoint to $M \odot$ - is called "right-hom", or "source-hom", and denoted $\operatorname{sHom}(M,-)$. The adjoint to $-\odot M$ is called "left-hom", or "target-hom", and denoted $t \operatorname{Hom}(M,-)$. For $M \in$ $\mathscr{B}(A, B)$ and $W \in \mathscr{B}(A, C)$, we have the 1-cell $\operatorname{sHom}(M, W) \in \mathscr{B}(B, C)$. For $U \in \mathscr{B}(D, B)$, we have the 1-cell $t \operatorname{Hom}(M, U) \in \mathscr{B}(D, A)$. The adjunctions are written as

$$
\begin{aligned}
\mathscr{B}(M \odot V, W) & \cong \mathscr{B}(V, \operatorname{sHom}(M, W)) \\
\mathscr{B}(T \odot M, U) & \cong \mathscr{B}(T, t \operatorname{Hom}(M, U))
\end{aligned}
$$

The existence of left and right hom functors defines a closed bicategory. A thorough description of closed structures can be found in [MS06].

3.5. Duality and invertibility in bicategories. For general discussion about duality, we consider fixed 1-cells $X: A \rightarrow B$ and $Y: B \rightarrow A$ in a closed bicategory $\mathscr{B}$.

Definition 3.3 (Dual pair). We say $(X, Y)$ is a dual pair, or " $X$ is left-dual to $Y$ " (" $Y$ is right-dual to $X$ "), or " $X$ is right-dualizable" (" $Y$ is left-dualizable") to mean that we have 2-cells

$$
\eta: A \rightarrow X \odot Y \text { and } \varepsilon: Y \odot X \rightarrow B
$$

such that the following composites are the respective identity 2-cells. These are known as the triangle identities:

$$
\begin{aligned}
& X \cong A \odot X \stackrel{\eta \odot \mathrm{id}}{\longrightarrow} X \odot Y \odot X \stackrel{\mathrm{id} \odot \varepsilon}{\longrightarrow} X \odot B \cong X \\
& Y \cong Y \odot A \stackrel{\text { id } \odot \eta}{\longrightarrow} Y \odot X \odot Y \stackrel{\varepsilon \odot \text { id }}{\longrightarrow} B \odot Y \cong Y .
\end{aligned}
$$


Lemma 3.4. Let $k$ be the unit of a symmetric monoidal category, and $A$ a $k$-algebra. Let ${ }_{k} A, A_{k}$ denote the base-change 1-cells induced by the unit map $k \rightarrow A$. Then $\left({ }_{k} A, A_{k}\right)$ is a dual pair with structure maps

$$
k \rightarrow{ }_{k} A \otimes_{A} A_{k}
$$

and

$$
A_{k} \otimes_{k} A \rightarrow A
$$

Note. When $(X, Y)$ is a dual pair, we will occasionally refer to this by saying that $X$ is dualizable over $B$, since the unit condition often amounts to a finiteness of $X$ over $B$. When $(Y, X)$ is a dual pair, we will say that $X$ is dualizable over $A$. With this convention, the phrase "dualizable over" always references the target of the evaluation map, and uniquely determines whether we mean leftor right-dualizable.

Definition 3.5 (Invertible pair). A dual pair $(X, Y)$ is called invertible if the maps $\eta$ and $\varepsilon$ are isomorphisms. Equivalently, the adjoint pairs described in Proposition 3.7 are adjoint equivalences.

Duality for monoidal categories has been studied at length; one reference for duality in a bicategorical context is [MS06, §16.4], and there are surely others in the categorical literature. The definition of duality does not require $\mathscr{B}$ to be closed, but we will make use of the following basic facts about duality, some of which do require a closed structure on $\mathscr{B}$. The following two results can be found in [MS06, §16.4].

Proposition 3.6. A 1-cell $X \in \mathscr{B}(A, B)$ is right-dualizable if and only if the coevaluation

$$
\nu: X \odot t \operatorname{Hom}(X, B) \rightarrow t \operatorname{Hom}(X, X)
$$

is an isomorphism. Moreover, this is the case if and only if the map

$$
\nu_{Z}: X \odot t \operatorname{Hom}(X, Z) \rightarrow t \operatorname{Hom}(X, X \odot Z)
$$

is an isomorphism for all 1-cells $Z: B \rightarrow B$.

Proposition 3.7. Let $(X, Y)$ be a dual pair in $\mathscr{B}$, with $X: A \rightarrow B$ and $Y: B \rightarrow A$.

i. For any 0-cell $C$, we have two adjoint pairs of functors, with left adjoints written on top:

$$
\begin{aligned}
\mathscr{B}(C, A) & \underset{-\odot Y}{\stackrel{-\odot X}{\rightleftarrows}} \mathscr{B}(C, B) \\
\mathscr{B}(A, C) & \stackrel{Y \odot-}{\underset{X \odot-}{\rightleftarrows}} \mathscr{B}(B, C)
\end{aligned}
$$

The structure maps for the dual pair give the triangle identities necessary to show that the displayed functors are adjoint pairs.

ii. The right dual, $Y$, is canonically isomorphic to $t \operatorname{Hom}(X, B)$, and for any 1-cell $W: D \rightarrow B$, the coevaluation map $W \odot t \operatorname{Hom}(X, B) \rightarrow t \operatorname{Hom}(X, W)$ is an isomorphism.

iii. The left dual, $X$, is canonically isomorphic to $\operatorname{sHom}(Y, B)$, and for any 1-cell $U: A \rightarrow D^{\prime}$, the coevaluation map $\operatorname{sHom}(Y, A) \odot U \rightarrow \operatorname{sHom}(Y, U)$ is an isomorphism.

Lemma 3.8. Let $X: A \rightarrow B$ be a 1-cell in $\mathscr{B}(A, B)$. If $X$ is right-dualizable and the unit $A \rightarrow$ $t \operatorname{Hom}(X, X)$ is an isomorphism, then the evaluation $X \odot s \operatorname{Hom}(X, A) \rightarrow A$ is an isomorphism. Likewise, if $X$ is left-dualizable and the unit $B \rightarrow s \operatorname{Hom}(X, X)$ is an isomorphism, then the evaluation $t \operatorname{Hom}(X, B) \odot X \rightarrow B$ is an isomorphism. 
Proof. We prove the first statement, leaving the second as an exercise in opposites. Let $Y$ denote the right dual of $X$. Since $X$ is right-dualizable, $Y$ is left-dualizable and $X$ is isomorphic to the canonical left dual of $Y: X \cong \operatorname{sHom}(Y, B)$. The isomorphism $A \stackrel{\cong}{\rightrightarrows} t \operatorname{Hom}(X, X)$ implies that the unit for the duality is an isomorphism: $A \stackrel{\cong}{\rightrightarrows} X \odot Y$. Now we have the following commutative square:

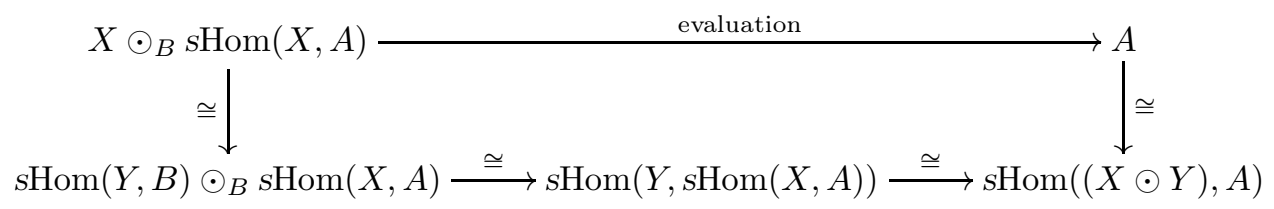

where the two vertical isomorphisms are described above, the left-hand isomorphism is a consequence of dualizability for $Y$, and the right-hand isomorphism is an exercise in adjunction.

3.6. Characterization of Azumaya objects. In this section we give a proof of Theorem 1.6 in two stages. Let $A$ be a fixed 0-cell of an Eilenberg-Watts bicategory $\mathscr{B}$.

Notation 3.9. Throughout, it will be useful to distinguish between the 0-cell $A$, the unit 1-cell $A: A \rightarrow A$ and the 1-cell $A: k \rightarrow A^{e}$ corresponding to $A: A \rightarrow A$ under the autonomy $\mathscr{B}(A, A) \simeq$ $\mathscr{B}\left(k, A^{e}\right)$. We will refer to the latter as the floor of $A$, and denote it as $\lfloor A\rfloor$, with the motivation that $\lfloor A\rfloor$ is naturally induced by $A$, but with its source being the ground ring and its target being the enveloping object, $A^{e}$. Thus $\lfloor A\rfloor$ is a 1 -cell with source $k$ and target $A^{e}$ :

$$
\lfloor A\rfloor: k \rightarrow A^{e} .
$$

We will not make explicit reference to the related 1-cell $A^{e} \rightarrow k$, but a compatible notation would be the ceiling, $\lceil A\rceil$. As for the 0-cell $A$ and corresponding unit 1-cell, these sit at different levels of the ambient bicategory, and this will be sufficient to distinguish them in context.

We now give the general characterization of Azumaya objects; Theorem 1.6 follows directly from this. First, we give natural generalizations of the classical terminology:

Definition 3.10 (Central/Endomorphic). We say that $A$ is central over $k$ if the natural unit map

$$
k \rightarrow t \operatorname{Hom}(\lfloor A\rfloor,\lfloor A\rfloor)
$$

is an isomorphism.

We say that $A$ is endomorphic over $k$ if the natural unit map

$$
A^{e} \rightarrow \operatorname{sHom}(\lfloor A\rfloor,\lfloor A\rfloor)
$$

is an isomorphism.

Definition 3.11 (Faithfully Projective/Faithfully Separable). We say that $A$ is faithfully projective over $k$ if both the coevaluation

$$
\operatorname{sHom}(\lfloor A\rfloor, k) \wedge_{k}\lfloor A\rfloor \rightarrow \operatorname{sHom}(\lfloor A\rfloor,\lfloor A\rfloor)
$$

and the evaluation

$$
\lfloor A\rfloor \wedge_{A^{e}} \operatorname{SHom}(\lfloor A\rfloor, k) \rightarrow k
$$

are isomorphisms.

We say that $A$ is faithfully separable over $k$ if both the coevaluation

$$
\lfloor A\rfloor \wedge_{A^{e}} t \operatorname{Hom}\left(\lfloor A\rfloor, A^{e}\right) \rightarrow t \operatorname{Hom}(\lfloor A\rfloor,\lfloor A\rfloor)
$$


and the evaluation

$$
t \operatorname{Hom}\left(\lfloor A\rfloor, A^{e}\right) \wedge_{k}\lfloor A\rfloor \rightarrow A^{e}
$$

are isomorphisms.

We are now ready to prove the characterization theorem for Azumaya objects in Eilenberg-Watts bicategories.

Proof of Theorem 1.6. In general, a pair of 1-cells $(X, Y)$ is invertible if and only if $X$ is rightdualizable, $Y$ is isomorphic to the canonical right dual of $X$, and both the unit and counit of the duality are isomorphisms. This gives the equivalence of $[$ and iii Likewise, $(Y, X)$ is invertible if and only if $X$ is left-dualizable, $Y$ is isomorphic to the canonical left dual of $X$, and both the unit and counit of the duality are isomorphisms. This gives the equivalence of $i$ and iv since $(X, Y)$ is an invertible pair if and only if $(Y, X)$ is such, the first three conditions are seen to be equivalent.

Connecting the last two conditions to the previous ones is essentially a bicategorical diagram chase: of course if $\lfloor A\rfloor$ is invertible, then the implication of the last two conditions is clear. For the reverse, suppose that $P: k \rightarrow A \otimes B$ is an invertible 1-cell between $k$ and $A \otimes B$ and let $P^{*}$ be its inverse. Let $P^{e}=P \otimes\left(P^{*}\right)^{\text {op }}$ and $P^{* e}=P^{*} \otimes P^{\text {op }}$. Then $\left(P^{e}, P^{* e}\right)$ is an invertible pair of 1-cells between the 0-cells $k^{e}$ and $(A \otimes B)^{e} \cong A^{e} \otimes B^{e}$ (using the symmetry). Moreover, since $\left(P, P^{*}\right)$ is an invertible pair we have $P^{e}=P \otimes\left(P^{*}\right)^{\text {op }} \cong\lfloor A \otimes B\rfloor \cong\lfloor A\rfloor \otimes\lfloor B\rfloor$ (again using symmetry). The diagram below describes our situation:

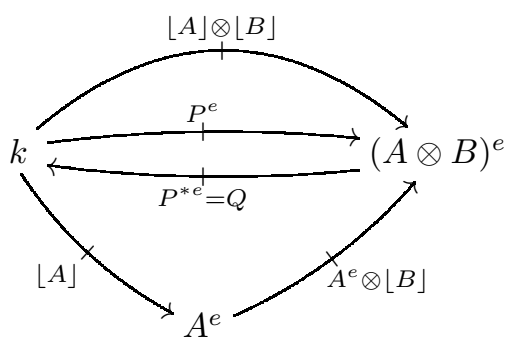

Let $Q=P^{* e}$ and let $T=\left(A^{e} \otimes\lfloor B\rfloor\right) \odot Q$. We have shown that $(\lfloor A\rfloor \otimes\lfloor B\rfloor, Q)$ is an invertible pair of 1-cells, and we will now show that $(\lfloor A\rfloor, T)$ is an invertible pair between $A^{e}$ and $k$. A straightforward use of associativity shows that $\lfloor A\rfloor \odot T \cong k$. We now use Lemma 3.2 for the reverse composite. This shows that $\lfloor A\rfloor$ is invertible, with the triangle identities coming from those of $\left(P^{e}, P^{* e}\right)$ :

$$
\begin{aligned}
T \odot\lfloor A\rfloor=\left(A^{e} \otimes\lfloor B\rfloor\right) \odot Q \odot\lfloor A\rfloor & \cong\left(A^{e} \otimes\lfloor B\rfloor\right) \odot\left(\lfloor A\rfloor \otimes A^{e} \otimes B^{e}\right) \odot\left(A^{e} \otimes Q\right) \\
& \cong\left(A^{e} \otimes\lfloor B\rfloor\right) \odot\left(A^{e} \otimes\lfloor A\rfloor \otimes B^{e}\right) \odot\left(A^{e} \otimes Q\right) \\
& \cong\left(A^{e} \otimes\lfloor A\rfloor \otimes\lfloor B\rfloor\right) \odot\left(A^{e} \otimes Q\right) \\
& \cong\left(A^{e} \otimes k\right) \cong A^{e} .
\end{aligned}
$$

\section{Triangulated Bicategories}

We recall first the definitions of localizing subcategory and generator for a triangulated category, and then give a definition (4.4) of triangulated bicategory suitable for our purposes. In particular, under this definition $\mathscr{D}_{k}$ is a triangulated bicategory when $k$ is a commutative d.g. ring or ring spectrum. 
Definition 4.1 (Localizing subcategory). If $\mathscr{T}$ is a triangulated category with infinite coproducts, a localizing subcategory, $\mathscr{S}$, is a full triangulated subcategory of $\mathscr{T}$ which is closed under coproducts from $\mathscr{T}$.

Remark 4.2. This is equivalent to the definition for arbitrary triangulated categories of [Hov99], (which requires that a localizing subcategory be thick) because a triangulated subcategory automatically satisfies the 2-out-of-3 property and because in any triangulated category with countable coproducts, idempotents have splittings. See [Nee01, 1.5.2, 1.6.8, and 3.2.7] for details.

Definition 4.3 (Triangulated generator). A set, $\mathscr{P}$, of objects in $\mathscr{T}$ (triangulated category with infinite coproducts, as above) is a set of triangulated generators (or simply generators) if the only localizing subcategory containing $\mathscr{P}$ is $\mathscr{T}$ itself.

Definition 4.4 (Triangulated bicategory [MS06, §16.7]). A closed bicategory $\mathscr{B}$ will be called a triangulated bicategory if for each pair of 0-cells, $A$ and $B, \mathscr{B}(A, B)$ is a triangulated category with infinite coproducts, and furthermore the local triangulations on $\mathscr{B}$ are compatible as described in the following axioms.

(TC0) The local suspension functors

$$
\Sigma_{A, B}: \mathscr{B}(A, B) \stackrel{\simeq}{\rightrightarrows} \mathscr{B}(A, B)
$$

assemble as the components of a strong transformation

$$
\Sigma: \mathscr{B}(-,-) \rightarrow \mathscr{B}(-,-) \text {. }
$$

This implies, in particular, that for a 1-cell $X: A \rightarrow B$ there are natural structure isomorphisms

$$
\Sigma A \odot X \stackrel{\alpha_{X}}{\cong} \Sigma(A \odot X) \cong \Sigma X \cong \Sigma(X \odot B) \stackrel{\beta_{X}}{\cong} X \odot \Sigma B .
$$

(TC1) For a 0-cell $A$, the following composite of structure isomorphisms interchanging suspension coordinates is multiplication by -1 :

$$
\Sigma^{2} A \stackrel{\Sigma \beta_{A}^{-1}}{\longrightarrow} \Sigma(A \odot \Sigma A) \stackrel{\alpha_{\Sigma A}^{-1}}{\longrightarrow} \Sigma A \odot \Sigma A \stackrel{\beta_{\Sigma A}}{\longrightarrow} \Sigma(\Sigma A \odot A) \stackrel{\Sigma \alpha_{A}}{\longrightarrow} \Sigma^{2} A .
$$

(TC2) Each of $-\odot-, s \operatorname{Hom}(-,-)$, and $t \operatorname{Hom}(-,-)$ is exact in both variables.

The axioms above generalize those of a triangulated monoidal category although, because we do not need it, we do not include a version of the braid axiom (TC3).

Remark 4.5. For a commutative d.g. algebra or ring spectrum $k$, each local category $\mathscr{D}_{k}(A, B)$ is triangulated, with the suspension functor given by horizontal composition $\Sigma A \odot-$. The monoidal category $\mathscr{D}_{k}(k, k)$ is well-known to satisfy the compatibility conditions, and the same reasoning shows that $\mathscr{D}_{k}$ satisfies these conditions in general.

If $\mathscr{B}$ is a triangulated bicategory and $P, Q$ are 1 -cells in $\mathscr{B}(A, B)$, we emphasize that $\mathscr{B}$ is triangulated by writing the abelian group of 2-cells $P \rightarrow Q$ as $\mathscr{B}[P, Q]$ and by writing the graded abelian group obtained by taking shifts of $Q$ as $\mathscr{B}[P, Q]_{*}$. To emphasize the source and target of $P$ and $Q$, we may also write $\mathscr{B}(A, B)[P, Q]_{*}$.

Definition 4.6 ( $\odot$-faithful 1-cells). In any locally additive bicategory, $\mathscr{B}$, a 1-cell $W: A \mapsto B$ is called source-faithful if triviality for any 1-cell $Z: C \rightarrow A$ is detected by triviality of the composite $Z \odot W$. That is, $Z: C \rightarrow A$ is zero if and only if $Z \odot W=0$. A collection of 1-cells, $\mathscr{E}$, in $\mathscr{B}(A, B)$ 
is called jointly source-faithful if the objects have this property jointly; that is, $Z=0$ if and only if $Z \odot W=0$ for all $W \in \mathscr{E}$. The term target-faithful is defined similarly, considering $W \odot-$ instead of $-\odot W$.

Remark 4.7. If $\mathscr{C}$ is an additive monoidal category with monoidal product $\odot$, the unit object is both source- and target-faithful. In an arbitrary locally additive bicategory $\mathscr{B}$, if $A \neq B$ then $\mathscr{B}(A, B)$ may not have a single object with this property. In relevant examples, however, the collection of all 1-cells, ob $\mathscr{B}(A, B)$, does have this property jointly.

Lemma 4.8. Let $\mathscr{B}$ be a triangulated bicategory, and let $P: A \rightarrow B$ be a generator for $\mathscr{B}(A, B)$. If the collection of all 1-cells, $\mathscr{B}(A, B)$, is jointly source-faithful (resp. target-faithful), then $P$ is source-faithful (resp. target-faithful).

Proof. Consider the source-faithful case; the target-faithful case is similar. Given any 1-cell

$$
Z: C \rightarrow A
$$

with $Z \odot P=0$, let $\mathscr{S}$ be the full subcategory of 1-cells, $W: A \rightarrow B$ for which $Z \odot W=0$. This is a localizing subcategory of $\mathscr{B}(A, B)$, and by assumption $P \in \mathscr{S}$, so $\mathscr{S}=\mathscr{B}(A, B)$, and hence $Z=0$.

Remark 4.9. Since the functors $P \odot-$ are exact, the property of $P \odot-$ detecting trivial objects is equivalent to $P \odot-$ detecting isomorphisms (meaning that a 2-cell $f$ is an isomorphism if and only if $P \odot f$ is so).

4.1. Homotopy Bicategories. In this section, let $\mathscr{C}$ be a complete and cocomplete closed symmetric monoidal model category. Let $\mathscr{M}$ be the closed bicategory formed by $\mathscr{C}$-monoids and their bimodules as in Example 2.7. We say that $\mathscr{M}$ has a local model structure if each category of $1-$ and 2-cells $\mathscr{M}(A, B)$ is a model category.

We now describe further conditions for the model structure on $\mathscr{C}$ which ensure that $\mathscr{M}$ has a local model structure and that the collection of homotopy categories assembles to an EilenbergWatts bicategory. The first of these, pushout products, implies that the horizontal composition of 1-cells descends to a homotopy bicategory. The second, unit replacement, implies that horizontal composition of 1-cells on the level of homotopy is unital.

Definition 4.10 (Pushout products). Suppose $\mathscr{M}$ has a local model structure. We say $\mathscr{M}$ has pushout products if, for any 0-cells $A, B$, and $C$ and for any cofibrations $f: U \rightarrow V$ in $\mathscr{M}(A, C)$ and $g: W \rightarrow X$ in $\mathscr{M}(C, B)$, the pushout product $f \square g$ below is a cofibration in $\mathscr{M}(A, B)$ which is acyclic if either $f$ or $g$ is acyclic.

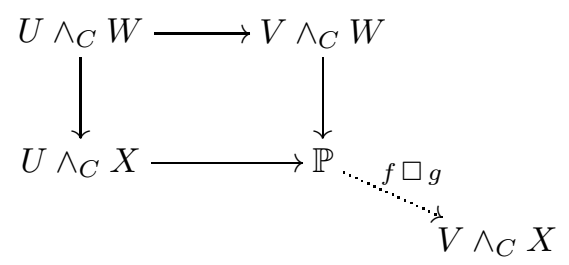

Definition 4.11 (Unit replacement condition). Let $A$ be a 0 -cell of $\mathscr{M}$, and let $Q A$ be a cofibrant replacement for the unit 1-cell $A$ in $\mathscr{M}(A, A)$. We say that the unit replacement condition holds for $A$ if, for any cofibrant $X \in \mathscr{M}(A, C)$, the induced map

$$
Q A \odot X \rightarrow A \odot X \cong X
$$


is a weak equivalence in $\mathscr{M}(A, C)$. Note that this condition is independent of the choice of cofibrant replacement $Q A$, and is automatically satisfied if $A$ is cofibrant in $\mathscr{B}(A, A)$.

Next we recall one result which implies that $\mathscr{M}$ has a local model structure, and then give another showing that this local model structure indeed descends to form a homotopy bicategory.

Proposition 4.12 ([SS00, 4.1]). Let $\mathscr{C}=(\mathscr{C}, \wedge, k)$ be a cofibrantly generated closed monoidal model category which satisfies the monoid axiom and in which each object is small with respect to the whole category. Let $\mathscr{M}_{\mathscr{C}}$ be the bicategory formed by $\mathscr{C}$-monoids which are cofibrant in $\mathscr{C}$ and their bimodules. Then $\mathscr{M}_{\mathscr{C}}$ has a local model structure, and has pushout products. Moreover, the category of $\mathscr{C}$-monoids $\operatorname{Mon}(\mathscr{C})$ is a cofibrantly generated model category, and if $k$ is cofibrant then every cofibration in $\operatorname{Mon}(\mathscr{C})$ whose source is cofibrant is also a cofibration upon forgetting to $\mathscr{C}$.

This proposition applies, for example, to simplicial sets, $\Gamma$-spaces, symmetric spectra, simplicial abelian groups, chain complexes, and $S$-modules. Details for these and other examples are given in [SS00, §5].

Proposition 4.13 (Homotopy bicategory). Let $\mathscr{C}$ be as above, and assume the following:

- The unit, $k$, is cofibrant in $\mathscr{C}$.

- The unit replacement condition holds for each monoid in $\mathscr{C}$ which is cofibrant in $\mathscr{C}$.

- For each monoid $A$ and cofibrant $A$-module $N,-\wedge_{A} N$ takes weak equivalences of $A$-modules to weak equivalences in $\mathscr{C}$.

Then the collection of homotopy categories $h \mathscr{M}_{\mathscr{C}}(A, B)$ forms a closed bicategory, which we denote h $\mathscr{M}_{\mathscr{C}}$.

Proof. We begin with an application of the theory of Quillen functors of two variables [Hov99, §4.2]. Since $\mathscr{M}_{\mathscr{C}}$ has pushout products by Proposition 4.12, each component of horizontal composition is a left Quillen bifunctor

$$
\odot: \mathscr{M}(A, C) \times \mathscr{M}(C, B) \rightarrow \mathscr{M}(A, B)
$$

and thus induces a bifunctor on $h \mathscr{M}$ which we also denote with $\odot$. The unit replacement condition ensures that $\odot$ is unital on $\mathscr{M}_{\mathscr{C}}$, and the third condition ensures that $h \mathscr{M}_{\mathscr{C}}(A, B) \simeq h \mathscr{M}_{\mathscr{C}}\left(A^{\prime}, B^{\prime}\right)$ if $A \simeq A^{\prime}$ and $B \simeq B^{\prime}$ are weak equivalences in $\operatorname{Mon}(\mathscr{C})$ [SS00, Theorem 3.3].

Proposition 4.14. Let $\mathscr{C}$ be as in Proposition 4.13. Then the derived monoidal product on $\mathscr{C}$

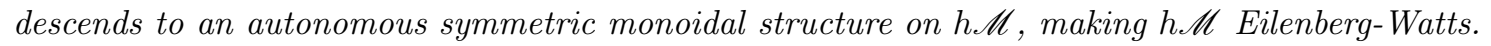

Proof. If $A$ and $B$ are cofibrant in $\mathscr{C}$, then $A \wedge B$ is a model for the derived product, and a cofibrant $(A, B)$-bimodule is also cofibrant in $\mathscr{C}$. Moreover, the product in $\mathscr{C}$ preserves bimodule structure and hence induces a bifunctor

$$
\wedge: h \mathscr{M}_{\mathscr{C}}(A, B) \times h \mathscr{M}_{\mathscr{C}}(C, D) \rightarrow h \mathscr{M}_{\mathscr{C}}(A \wedge C, B \wedge D)
$$

which agrees (for each $A, B, C, D \in \operatorname{Mon}(\mathscr{C})$ ) with the derived monoidal product in $\mathscr{C}$. This gives an associative product on the bicategory $h \mathscr{M}_{\mathscr{C}}$, which is unital because $k$ is cofibrant in $\mathscr{C}$. The autonomous structure on $h \mathscr{M}_{\mathscr{C}}$ is obtained by taking the opposite multiplication on monoids and modules of $\mathscr{C}$, and the symmetry of the product in $\mathscr{C}$ gives the symmetry in $h \mathscr{M}_{\mathscr{C}}$.

We now give our main application, in which $k$ is taken to be a commutative d.g. algebra or commutative ring spectrum and $\mathscr{C}=\mathscr{C}_{k}$ is taken to be the category of $k$-modules. One may work with any monoidal model category of spectra, but we must assume that $k$ is cofibrant in $\mathscr{C}_{k}$ and thus the result applies only to those categories in which the unit is cofibrant (e.g., symmetric spectra or 
orthogonal spectra). Likewise, if $k$ is a d.g. algebra, one must choose a model structure on $\mathscr{C}_{k}$ for which the unit is cofibrant (e.g., the injective model structure).

Proposition 4.15. Let $k$ be a commutative d.g. algebra or commutative ring spectrum, and let $\mathscr{C}_{k}$ be the symmetric monoidal category of $k$-modules, with a model structure for which $k$ is cofibrant. Then we have Eilenberg-Watts bicategories $\mathscr{M}_{k}=\mathscr{M}_{\mathscr{C}_{k}}$ and $\mathscr{D}_{k}=h \mathscr{M}_{\mathscr{C}_{k}}$.

Proof. The hypotheses of Propositions 4.12 and 4.13 are satisfied by $\mathscr{C}_{k}$, so Proposition 4.14 applies: most of the hypotheses are discussed in [SS00] and so we comment only on the unit replacement condition. Suppose $A \in \operatorname{Mon}_{c}(\mathscr{C})$, and consider a specific cofibrant replacement for $A$ as an $(A, A)$ bimodule: the two-sided bar construction $Q A=B(A, A, A)$. Then $Q A \wedge_{A} X \simeq B(A, A, X)$, and the latter retracts to $A \wedge_{A} X$ along the simplicial retraction induced by the unit of $A$. Thus the induced map

$$
Q A \wedge_{A} X \rightarrow A \wedge_{A} X \simeq X
$$

is an equivalence in $\mathscr{D}_{k}(A, A)$.

4.2. Invertibility in triangulated bicategories. For this section, we let $\mathscr{D}$ denote a triangulated Eilenberg-Watts bicategory with unit 0-cell $k$. Bousfield localization for triangulated categories [Kra10, §4.10] generalizes to triangulated bicategories in two ways, using 1-cell composition over either the source or target of a given 1-cell. Recall that we write 1-cell composition in diagrammatic order:

$$
(C \stackrel{M}{\rightarrow} A) \odot(A \stackrel{T}{\rightarrow} B)=(C \stackrel{M \odot T}{\longrightarrow} B)
$$

Definition 4.16. Let $T: A \rightarrow B$ be a 1-cell in $\mathscr{D}(A, B)$.

A 1-cell $M: C \mapsto A$ is source- $T$-acyclic if $M \odot T=0$. A 1-cell $N: C \rightarrow A$ is source-T-local if

$$
\mathscr{D}(C, A)[M, N]_{*}=0
$$

for all source- $T$-acyclic 1-cells $M \in \mathscr{D}(C, A)$. The full subcategory of source-T-local 1-cells in $\mathscr{D}(C, A)$ is denoted $\mathscr{D}(C, A)_{\langle\odot T\rangle}$.

A 1-cell $M^{\prime}: B \rightarrow C$ is target-T-acyclic if $T \odot M=0$. A 1-cell $N^{\prime}: B \rightarrow C$ is target-T-local if

$$
\mathscr{D}(B, C)\left[M^{\prime}, N^{\prime}\right]_{*}=0
$$

for all target- $T$-acyclic 1-cells $M^{\prime} \in \mathscr{D}(B, C)$. The full subcategory of target-T-local 1-cells in $\mathscr{D}(B, C)$ is denoted $\mathscr{D}(B, C)_{\langle T \odot\rangle}$.

Baker and Lazarev describe the following in the context of spectra, but their methods generalize to our setting. The key observation is that for any 1-cell $P$ whose source is $A, \operatorname{sHom}(T, P)$ is target- $T$-local. Likewise, if $P^{\prime}$ is any 1-cell whose target is $B, t \operatorname{Hom}\left(T, P^{\prime}\right)$ is source- $T$-local.

Proposition 4.17 ([BL04]). Let $T: A \rightarrow B$ be a 1-cell in $\mathscr{D}(A, B)$. The adjunctions induced by $T$ factor through the T-local pseudofunctors, which is to say that we have the following diagrams of adjoint transformations:
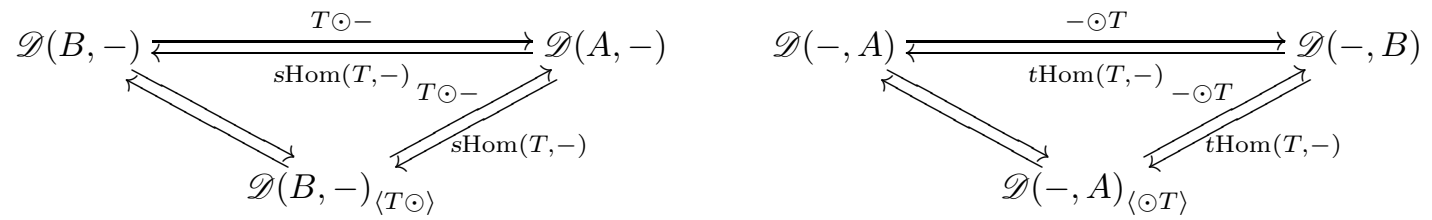
Proposition 4.18 ([BL04). If a 1-cell $T \in \mathscr{D}(A, B)$ is right-dualizable and the unit map induces an isomorphism $A \cong t \operatorname{Hom}(T, T)$, then the induced adjoint pair is an equivalence

$$
\mathscr{D}(B,-)_{\langle T \odot\rangle} \simeq \mathscr{D}(A,-) .
$$

We have a corresponding statement for the case of left-dualizability.

Proof. It follows immediately from Lemma 3.8 that the evaluation map

$$
T \odot \operatorname{sHom}(T, M) \rightarrow M
$$

is an isomorphism for all $M$; this is the counit of the adjunction. Moreover, $s \operatorname{Hom}(T,-)$ takes values in the target- $T$-local category and hence the fact that the unit of the adjunction is an isomorphism follows from the fact that the counit is so.

Corollary 4.19. Let $T: A \rightarrow B$ be as in Proposition 4.18. Then $T$ is target-faithful (Definition 4.6) if and only if localization induces an equivalence between the category of 1-cells $\mathscr{B}(B, B)$ and the target-T-local subcategory. In this case each of the three adjoint pairs of Proposition 4.17 (at left) is an equivalence. We have a corresponding statement for the source-faithful case.

Proof. If $T$ is target-faithful, then all target- $T$-acyclics are trivial, and therefore

$$
\mathscr{D}(B,-) \simeq \mathscr{D}(B,-)_{\langle T \odot\rangle} .
$$

The statement then follows from Proposition 4.18. Conversely, if localization induces an equivalence $\mathscr{D}(B, B) \simeq \mathscr{D}(B, B)_{\langle T \odot\rangle}$ then the proof of Proposition 4.18 shows that $T$ is invertible and hence target-faithful.

Definition 4.20. We say that $B$ is strongly target-T-local if localization induces an equivalence

$$
\mathscr{D}(B, B) \simeq \mathscr{D}(B, B)_{\langle T \odot\rangle} .
$$

Likewise, we say that $A$ is strongly source-T-local if localization induces an equivalence

$$
\mathscr{D}(A, A) \simeq \mathscr{D}(A, A)_{\langle\odot T\rangle} .
$$

Combining the previous results yields a characterization of invertible objects in triangulated Eilenberg-Watts bicategories. Applying this to the case $T=\lfloor A\rfloor$ immediately gives the characterization of Azumaya objects in Theorem 1.8.

Proposition 4.21. Let $T: A \rightarrow B$ be a 1-cell in $\mathscr{D}$. The following are equivalent:

i. $T$ is invertible.

ii. a) $T$ is right-dualizable.

b) The unit induces $A \cong t \operatorname{Hom}(T, T)$.

c) $B$ is strongly target-T-local.

iii. a) $T$ is left-dualizable.

b) The unit induces $B \cong \mathrm{sHom}(T, T)$.

c) $A$ is strongly source-T-local.

Remark 4.22. We have the following chain of implications for $T: A \mapsto B$, and a similar chain for source-faithful and source-local conditions:

$T$ is target-faithful. $\Rightarrow B$ is strongly target- $T$-local. $\Rightarrow B$ is target- $T$-local.

Corollary 4.19 shows that the first implication is an equivalence under the conditions of Proposition 4.18, and therefore condition $(c)$ in each of the statements of Proposition 4.21 can be replaced by the corresponding faithfulness condition on $T$. 
4.3. Application to tilting theory. The work in this section allows us to give a unified proof of results from the tilting theory of [Ric89] and [SS03b]:

Proof of 1.12 and 1.13. Let $\widetilde{T}$ denote $T$ regarded as a bimodule over $A=t \operatorname{Hom}(T, T)$. Since $T$ is right-dualizable, $\widetilde{T}$ is right-dualizable in $\mathscr{D}_{k}(A, B)$. Moreover, $A \simeq t \operatorname{Hom}(\widetilde{T}, \widetilde{T})$.

By Remark 4.5 and Proposition 4.15, $\mathscr{D}_{k}$ is a triangulated Eilenberg-Watts bicategory. Since $k$ is the unit of $\mathscr{D}_{k}$, the 1-cells of $\mathscr{D}_{k}(k, B)$ are jointly target-faithful (Definition 4.6 ). Since $T$ generates $\mathscr{D}_{k}(k, B)$, Lemma 4.8 shows that $T$ is target-faithful and this means that $\widetilde{T}$ is also target-faithful. The result then follows from Corollary 4.19 and Proposition 4.21 .

\section{Homotopical Brauer Groups}

This section describes homotopical Brauer groups for rings and ring spectra; these constitute our main applications of the preceding theory. We also give explicit comparisons between the Brauer group as characterized by Theorems 1.6 and 1.8 and as it appears in related work on Brauer groups in homotopical settings. We begin with the derived Brauer group of a ring and then address the Brauer groups of ring spectra.

\subsection{The derived Brauer group of a ring.}

Definition 5.1. We refer to $\operatorname{Br}\left(\mathscr{D}_{k}\right)$ as the derived Brauer group of $k$, to distinguish it from the generally different classical Brauer group, $B r(k)$.

Toën Toë12 introduces the notion of derived Azumaya algebras (the two conditions appearing in Proposition 5.2), and describes a Brauer group formed by Eilenberg-Watts equivalence classes of such. We show that the notion of derived Azumaya algebra is equivalent to the notion of Azumaya object in the derived category, and therefore the resulting Brauer groups are isomorphic.

Proposition 5.2. Let $k$ be a graded commutative ring and let $A$ be a $k$-algebra, regarded as a left module over $k$ and a right module over the enveloping algebra $A^{e}$. Then $A$ is an Azumaya object of $\mathscr{D}_{k}$ if and only if the following two conditions hold:

$i$. The underlying $k$-module of $A$ is a compact generator of the triangulated category $\mathscr{D}_{k}(k, k)$.

ii. The map $\mu: A^{e} \rightarrow F_{k}(A, A)$ is an equivalence.

Proof. Toën [Toë12, 2.8] shows that the conditions above are equivalent to the condition that $A^{e}$ be Eilenberg-Watts equivalent to $k$ in the bicategory $\mathscr{D}_{k}$, and this is one of the conditions appearing in the characterization Theorem 1.6.

Combining this with [Toë12, 2.12] we have the following:

Proposition 5.3. Let $k$ be a field. Then $\operatorname{Br}\left(\mathscr{D}_{k}\right)$ is isomorphic to the classical Brauer group $\operatorname{Br}(k)$.

For $k$ not a field, this result does not generalize, although there is of course always a homomorphism

$$
\operatorname{Br}(k) \rightarrow \operatorname{Br}\left(\mathscr{D}_{k}\right) .
$$

5.2. The Brauer group of a ring spectrum. When $k$ is a commutative ring spectrum Baker, Richter and Szymik have introduced and studied the notion of topological Azumaya $k$-algebra BRS12. Their definition (the three conditions of Proposition 5.4 below) makes sense in any modern monoidal model category of spectra, and we show it is equivalent to the definition of Azumaya used here. Since the various modern categories of spectra are all strong monoidal Quillen equivalent SS03a, the results of homotopical Brauer theory transfer between any of them. 
Recall that $\lfloor A\rfloor: k \rightarrow A^{e}$ denotes $A$ regarded as a right module over $A^{e}$, and ${ }_{k} A_{k}=\lfloor A\rfloor_{k}$ is the underlying $(k, k)$-module of $A$.

Proposition 5.4. Let $k$ be a commutative ring spectrum such that $\mathscr{D}_{k}$ has the structure of a triangulated Eilenberg-Watts bicategory. Let $A$ be a $k$-algebra, regarded as a left module over $k$ and a right module over the enveloping algebra $A^{e}$. Then $A$ is an Azumaya object in $\mathscr{D}_{k}$ if and only if the following conditions hold:

i. A is dualizable in the homotopy category of $k$-modules.

ii. The map $A^{e} \rightarrow F_{k}(A, A)$ is a weak equivalence of bimodules over $A^{e}$.

iii. A is faithful as a $k$-module.

Proof. Baker, Richter, and Szymik show that these conditions imply that $\lfloor A\rfloor$ is central and separable over $k$ [BRS12, 1.3, 1.4]. They are working with $S$-algebras, but those results apply equally well in any modern category of spectra. Now if $\lfloor A\rfloor_{k}=\lfloor A\rfloor \odot A_{k}$ is faithful, then $\lfloor A\rfloor$ must be targetfaithful (Definition 4.6). Remark 4.22 explains that under these circumstances target-faithfulness of $\lfloor A\rfloor$ is equivalent to $A^{e}$ being strongly target- $\lfloor A\rfloor$-local, and thus $A$ is Azumaya by Proposition 4.21 .

For the converse, we again refer to Proposition 4.21 . Lemma 3.4 shows that $\lfloor A\rfloor$ being leftdualizable implies $\lfloor A\rfloor_{k}$ is left-dualizable, and under these circumstances $k$ being strongly source$\lfloor A\rfloor$-local is equivalent to $\lfloor A\rfloor$ being source-faithful (Remark 4.22). Since restriction of scalars along the unit map $k \rightarrow A^{e}$ is faithful, the composite $\lfloor A\rfloor_{k}$ must also be faithful.

One minor subtlety remains in the choice of equivalence relation with which we form a Brauer group. There are two standard choices, Eilenberg-Watts equivalence and Brauer equivalence. We finish by showing that the two resulting groups are isomorphic. This is a classical fact for discrete rings which holds for formal reasons.

Definition 5.5 (Brauer equivalence). Two $k$-algebras $A_{1}$ and $A_{2}$ are called (topological) Brauer equivalent if there are faithful, dualizable, cofibrant $k$-modules $M_{1}$ and $M_{2}$ such that

$$
A_{1} \wedge_{k} F_{k}\left(M_{1}, M_{1}\right) \simeq A_{2} \wedge F_{k}\left(M_{2}, M_{2}\right)
$$

as $k$-algebras.

Proposition 5.6 ([BRS12, 2.4]). The collection of Azumaya k-algebras modulo Brauer equivalence forms a group.

Lemma 5.7. The group of Brauer equivalence classes of Azumaya $k$-algebras is isomorphic to the group of Eilenberg-Watts equivalence classes of such algebras.

Proof. For a dualizable $k$-module $M, F_{k}(M, M)$ is always Eilenberg-Watts equivalent to $k$. So if $A_{1}$ is Brauer equivalent to $A_{2}$ then it is Eilenberg-Watts equivalent to $A_{2}$. Therefore Brauer equivalence implies Eilenberg-Watts equivalence and there is a surjective group homomorphism from Azumaya algebras modulo Brauer equivalence to Azumaya algebras modulo Eilenberg-Watts equivalence. But if $A$ is Eilenberg-Watts equivalent to the ground ring, $k$, then there is an invertible bimodule $M$ giving the equivalence, and $A \simeq F_{k}(M, M)$, so $A$ is also Brauer equivalent to $k$. Thus this homomorphism is an isomorphism.

\section{REFERENCES}

[AG60] Maurice Auslander and Oscar Goldman, The Brauer group of a commutative ring, Transactions of the American Mathematical Society 97 (1960), no. 3, 367-409.

[AG12] B. Antieau and D. Gepner, Brauer groups and étale cohomology in derived algebraic geometry, arxiv:1210.0290, 2012. 
[Aus66] Maurice Auslander, The Brauer group of a ringed space, Journal of Algebra 4 (1966), $220-273$.

[Azu51] Gorô Azumaya, On maximally central algebras, Nagoya Math. J. 2 (1951), 119-150. MR MR0040287 $(12,669 \mathrm{~g})$

[BL04] A. Baker and A. Lazarev, Topological Hochschild cohomology and generalized Morita equivalence, Algebraic \& Geometric Topology 4 (2004), 623-645.

[BRS12] A. Baker, B. Richter, and M. Szymik, Brauer groups for commutative S-algebras, Journal of Pure and Applied Algebra 216 (2012), no. 11, 2361-2376.

[BV02] F. Borceux and E. Vitale, Azumaya Categories, Applied Categorical Structures 10 (2002), no. 5, $449-467$.

[Coh03] P. M. Cohn, Further algebra and applications, Springer-Verlag London Ltd., London, 2003.

[DI71] Frank DeMeyer and Edward Ingraham, Separable algebras over commutative rings, Lecture Notes in Mathematics, Vol. 181, Springer-Verlag, Berlin, 1971. MR MR0280479 (43 \#6199)

[DS97] B. Day and R. Street, Monoidal bicategories and Hopf algebroids, Advances in Mathematics 129 (1997), no. 1, 99-157.

[DS07a] D. Dugger and B. Shipley, Enriched model categories and an application to additive endomorphism spectra, Theory Appl. Categ. 18 (2007), No. 15, 400-439 (electronic). MR MR2342167 (2009d:18012)

[DS07b] _ Topological equivalences for differential graded algebras, Adv. Math. 212 (2007), no. 1, 37-61. MR MR2319762 (2008e:55025)

[DT12] I. Dell'Ambrogio and G. Tabuada, A Quillen model for classical Morita theory and a tensor categorification of the Brauer group, arxiv:1211.2309, 2012.

[EKMM97] A. D. Elmendorf, I. Kriz, M. A. Mandell, and J. P. May, Rings, modules, and algebras in stable homotopy theory, Mathematical Surveys and Monographs, vol. 47, American Mathematical Society, Providence, RI, 1997, With an appendix by M. Cole. MR MR1417719 (97h:55006)

[GO12] N. Gurski and A.M. Osorno, Infinite loop spaces, and coherence for symmetric monoidal bicategories, arxiv:1210.1174, 2012.

[GPS95] R. Gordon, A. J. Power, and Ross Street, Coherence for tricategories, vol. 117, Mem. Amer. Math. Soc., no. 558, Amer. Math. Soc., 1995. MR MR1261589 (96j:18002)

[Gro68] A. Grothendieck, Le groupe de Brauer. I - III, Dix Exposes sur la Cohomologie des Schemas (1968), 46-188.

[Gur11] N. Gurski, Loop spaces, and coherence for monoidal and braided monoidal bicategories, Advances in Mathematics 226 (2011), no. 5, 4225-4265.

[Hov99] M. Hovey, Model Categories, American Mathematical Society, 1999.

[Hov09] The Eilenberg-Watts theorem in homotopical algebra, arxiv:0910.3842, 2009.

[Joh08] N. Johnson, Morita theory for derived categories: A bicategorical perspective, arxiv:0805.3673 2008.

[KO74] M. A. Knus and M. Ojanguren, Théorie de la déscente et algébres d'Azumaya, Lecture Notes in Math, vol. 389, Springer-Verlag, Berlin-Heidelberg-Tokyo-New York, 1974.

[Kra10] Henning Krause, Localization theory for triangulated categories, Triangulated Categories, London Mathematical Society Lecture Note Series, no. 375, Cambridge University Press, 2010, pp. 161-235.

[KZ98] S. König and A. Zimmermann, Derived equivalences for group rings. With contributions by B. Keller, M. Linckelmann, J. Rickard and R. Rouquier, Lecture Notes in Mathematics, vol. 1685, Springer-Verlag, Berlin, 1998.

[MMSS01] M. A. Mandell, J. P. May, S. Schwede, and B. Shipley, Model categories of diagram spectra, Proceedings of the London Mathematical Society 82 (2001), no. 02, 441-512.

[MS06] J.P. May and J. Sigurdsson, Parametrized Homotopy Theory, Mathematical Surveys and Monographs, vol. 132, American Mathematical Society, 2006.

[Nee01] A. Neeman, Triangulated Categories, Princeton University Press, 2001.

[Par75] B. Pareigis, Non-additive ring and module theory IV: The Brauer group of a symmetric monoidal category, Brauer Groups (A. Dold and B. Eckmann, eds.), Lecture Notes in Mathematics, vol. 549, 1975.

[Ric89] J. Rickard, Morita Theory for Derived Categories, Journal of the London Mathematical Society 2 (1989), no. 3, 436 .

[Shi06] B. Shipley, Morita Theory In Stable Homotopy Theory, Handbook on Tilting Theory, London Math. Soc. Lecture Note Ser., vol. 332, Cambridge University Press, 2006, pp. 393-409.

[Shi07] _ HZ-algebra spectra are differential graded algebras, American Journal of Mathematics 129 (2007), no. 2, 351-379.

[SP09] C. Schommer-Pries, The classification of two-dimensional extended topological quantum field theories, Ph.D. thesis, UC Berkeley, 2009, arxiv:1112.1000 
[SS00] S. Schwede and B. Shipley, Algebras and modules in monoidal model categories, Proceedings of the London Mathematical Society 80 (2000), no. 2, 491.

[SS03a] _ Equivalences of monoidal model categories, Algebraic \& Geometric Topology 3 (2003), 287-334.

[SS03b] Stable model categories are categories of modules, Topology 42 (2003), no. 1, 103-153.

[Str03] R. Street, Functorial calculus in monoidal bicategories, Appl. Categ. Structures 11 (2003), no. 3, 219227. MR MR1990033 (2004f:18010)

[Toë12] B. Toën, Derived Azumaya algebras and generators for twisted derived categories, Inventiones Mathematicae 189 (2012), no. 3, 581-652.

[Vit96] E.M. Vitale, The Brauer and Brauer-Taylor groups of a symmetric monoidal category, Cahiers de Topologie et Géometrie Différentielle Catégoriques 37 (1996), 91-122.

[VOZ98] Fred Van Oystaeyen and Yinhuo Zhang, The Brauer group of a braided monoidal category, J. Algebra 202 (1998), no. 1, 96-128. MR MR1614178 (99c:18006)

$U R L:$ http://www.nilesjohnson.net

E-mail address: niles@math.ohio-state.edu

Dept. of Mathematics, The Ohio State University Newark, 1179 University Drive, Newark, OH 43055 USA 